University of Nebraska - Lincoln

DigitalCommons@University of Nebraska - Lincoln

2012

\title{
Government Insurance Program Design, Incentive Effects, and Technology Adoption: The Case of Skip-Row Crop Insurance
}

Joshua D. Woodard

Cornell University, joshua.woodard@cornell.edu

Alexander Pavlista

University of Nebraska-Lincoln, apavlista@unl.edu

Gary D. Schnitkey

University of Illinois at Urbana-Champaign, schnitke@illinois.edu

Paul Burgener

University of Nebraska-Lincoln, pburgener2@unl.edu

Kimberley A. Ward

Windsor Strategy Partners, kward0601@gmail.com

Follow this and additional works at: https://digitalcommons.unl.edu/panhandleresext

Part of the Agriculture Commons

Woodard, Joshua D.; Pavlista, Alexander; Schnitkey, Gary D.; Burgener, Paul; and Ward, Kimberley A., "Government Insurance Program Design, Incentive Effects, and Technology Adoption: The Case of SkipRow Crop Insurance" (2012). Panhandle Research and Extension Center. 58.

https://digitalcommons.unl.edu/panhandleresext/58

This Article is brought to you for free and open access by the Agricultural Research Division of IANR at DigitalCommons@University of Nebraska - Lincoln. It has been accepted for inclusion in Panhandle Research and Extension Center by an authorized administrator of DigitalCommons@University of Nebraska - Lincoln. 
Published in American Journal of Agricultural Economics 94:4 (2012), pp. 823-837; doi: 10.1093/ajae/aas018

Copyright (c) 2012 Joshua D. Woodard, Alexander D. Pavlista, Gary D. Schnitkey, Paul A. Burgener, and Kimberley A. Ward.

Published by Oxford University Press on behalf of the Agricultural and Applied Economics Association. Used by permission.

Portions of this work were derived from the USDA-RMA contracted actuarial study "Evaluation of Skip Row Corn and Grain Sorghum in the Central Great Plains", Solicitation AG-645S-S-09-0015. The authors would like to thank Maureen Flood, Ronald Lundine, and others at RMAUSDA, as well as Daniel Palic, two anonymous reviewers, and the editor of $A J A E$ for helpful comments and suggestions. All errors are our own.

Submitted January 2012; published online February 23, 2012.

\title{
Government Insurance Program Design, Incentive Effects, and Technology Adoption: The Case of Skip-Row Crop Insurance
}

\author{
Joshua D. Woodard, Alexander D. Pavlista, Gary D. Schnitkey, \\ Paul A. Burgener, and Kimberley A. Ward \\ Corresponding author - Joshua D. Woodard, tel 217 979-1251, fax 815 328-2252, email joshua.woodard@cornell.edu
}

\begin{abstract}
Joshua D. Woodard is an assistant professor in the Dyson School of Applied Economics and Management at Cornell University. Alexander D. Pavlista is a Professor of Agronomy \& Horticulture, University of Nebraska Panhandle Research and Extension Center. Gary D. Schnitkey is a Professor in the Department of Agricultural and Consumer Economics at the University of Illinois at Urbana-Champaign.

Paul A. Burgener is a Research Analyst at the Panhandle Research and Extension Center, University of Nebraska-Lincoln. Kimberley A. Ward is a Consulting Actuary for Windsor Strategy Partners
\end{abstract}

\begin{abstract}
Can the availability of poorly-designed government insurance alter technology adoption decisions? A theoretical model of technology adoption and insurance incentive effects for a high- and low-risk technology is developed and explored empirically using a unique dataset of skip-row agronomic trial data. A multivariate nonparametric resampling technique is developed, which augments the trial data with a larger dataset of conventional yields to improve estimation efficiency. Skip-row adoption is found to increase mean yields and reduce risk in areas prone to drought. RMA insurance rules have incentive-distorting impacts which disincentivize skip-row adoption.
\end{abstract}

Keywords: Adverse selection, federal Crop Insurance, insurance design, insurance pricing, nonparametric methods, risk management, skip-row corn, technology adoption, yield risk

\section{Introduction}

It has long been recognized that in the presence of information asymmetries, the availability of insurance can alter otherwise optimal behavior after the insurance purchase, and also that the availability of mispriced insurance can alter the type and amount of insurance purchased (see e.g. Arrow 1963; Rothschild and Stiglitz 1976; Akerlof 1970; Hölmstrom 1979). Although their roots are similar, the former is referred to as moral hazard, while the latter is referred to as adverse selection. The applications of these concepts to questions in insurance markets are numerous. However, one related question that has received less attention is how alternative government insurance market/product designs can impact the technology adoption decisions of producers in this context. This question is of great importance in crop insurance markets, as the provision of poorlydesigned government insurance may have the potential to disincentivize the adoption of otherwise optimal technologies, to the detriment of both the government and the adopters.

A particular market of interest in this respect is skip-row corn in the Central Great Plains under the Federal Crop Insurance Program. Producers of dryland crops in areas of low rainfall sometimes employ what are known as skip-row planting patterns; this is done by skipping rows when planting. Skiprow practices may better facilitate the maintenance of soil moisture throughout the growing season via inter-temporal rationing, as the crop root system is only able to reach the moisture in the skipped rows later in the growing season-rows which oth- 
erwise would have been exhausted if planted and not skipped. The crop physiological characteristics of skip-row suggest that there is a potential for this practice to outperform conventional fully-planted practices when moisture conditions are less than optimal, and vice-versa when conditions are favorable (see e.g. Lyon et al. 2009; Palic et al. 2008; Whish et al. 2005; Abunyewa 2008).

Skip-row patterns have been used historically in several regions and crops with some success (e.g. Texas cotton), and have recently gained some popularity in the Central Great Plains for corn (Pavlista et al. 2010) and grain sorghum (Abunyewa 2008). Yet the slow adoption of skip-row in the Central Great Plains has given rise to several questions regarding whether the practice is indeed beneficial from a production standpoint-in terms of impact on expected yields, yield risk, and net returns-and if so, how it should be properly treated in insurance contexts. The latter is quite important since the presence of the insurance market could have behavior-altering incentive effects regarding technology adoption decisions. If the insurance products are not properly designed and/or rated, or if the structure of the insurance market is such that one technology is favored over another in terms of coverage or relative pricing, then situations can arise where an otherwise optimal technology choice may be altered.

This study contributes to our understanding of the effects of government insurance programs on technology adoption. The paper also presents a novel empirical framework for integrating experimental trial data on new technologies with data from existing technologies in order to generate improved estimates of the production distribution of the new technology. To accomplish this, a theoretical model is first developed to investigate the impact of alternative government insurance program designs on technology adoption decisions using the case of skip-row corn in the Central Great Plains as a motivating application. Next, the skip-row rules from the Risk Management Agency (RMA) are investigated and reviewed; the RMA is charged with administering the Federal Crop Insurance Program. An empirical model is then developed to assess the characteristics of skiprow corn relative to conventional practices, focusing on both yield distribution impacts and implications for technology adoption. Specifically, three questions are addressed. First, in the absence of insurance, which technology (skip-row or conventional) would be optimal from the perspective of a risk-averse producer? Second, have the past and present RMA skiprow insurance rules crowded out skip-row adoption?
And third, are the production characteristics of the two practices substantially similar enough to warrant being combined as a single insurable practice as they are under current RMA rules, or should they be maintained as separate insurable practices in order to avoid incentive distortions that impact technology adoption?

Since skip-row practices are relatively new to the Central Great Plains, data are limited. To alleviate this problem, a unique dataset of side-by-side skip-row corn agronomic trial data $(N=270)$ are augmented with a large farm-unit level dataset consisting of 130,080 conventional corn yield observations from 1996-2008 by employing a novel multivariate nonparametric simulation technique to derive skiprow yield distributions and insurance rates. Applying this technique allows for a more efficient estimation of yield distributions in different locations under the new skip-row technology since the resulting estimates will embody a wider spectrum of possible weather events. To our knowledge, this is the first such attempt at exploiting this nature of side-by-side trial data on new crop technologies in order to obtain augmented yield distribution estimates. Using these simulated distributions, analysis of a representative producer is performed to evaluate the impacts of various insurance regimes on skip-row adoption.

We find that the optimal technology adoption decision depends critically on the design of the government's insurance market, and in extreme cases, a poorly-designed insurance system can completely crowd out the adoption of an otherwise optimal technology. Specifically, this study finds that the adoption of skip-row technologies results in substantial increases in expected yields in the regions and crops under consideration. The adoption of skip-row cropping also substantially limits the frequency and severity of yield losses, and results in fair premium reductions in excess of 50\% in typical cases. Empirical analysis suggests that current and former RMA skiprow insurance rules have incentive-distorting impacts that effectively crowd out skip-row adoption, suggesting that it may be more appropriate to treat skip-row cropping as a separate insurable practice as opposed to pooling the practices. Practical implementation considerations are also discussed.

\section{Theoretical Model - Insurance Design and Technol- ogy Adoption}

Following Rothschild and Stiglitz (1976), consider a risk-averse expected utility maximizing producer 
with utility function $U(x)$, where $x$ is end-of-period return, $U^{\prime}(x)>0$, and $U^{\prime \prime}(x)<0$. Suppose there are two technologies available, an existing high-risk technology and a newer low-risk technology, where $y_{E, L}$ and $y_{N, L}$ are the returns to the existing and new technologies, respectively, in the low-yielding state, both occurring simultaneously and with probability $p$, and $y_{E, H}$ and $y_{N, H}$ are the returns in the high-yielding state, occurring with probability $(1-p)$. Further assume that $y_{N, H}=y_{E, H}-r p /(1-p)$, and $y_{N, L}=y_{E, L}+\gamma$, where $\left(y_{E, H}-y_{E, L}\right) /[1+p /(1-p)]>\gamma>0$ holds, so that $y_{N, H}>y_{N, L}$. Thus, the new technology outperforms the existing technology in the low-yielding state, and underperforms in the high-yielding state. We assume the producer either adopts the new technology or not. It follows that

$$
\begin{aligned}
E\left(y_{N}\right) & =p y_{E, L}+(1-p) y_{E, H} \\
& =(1-p)\left(y_{E, H}-\gamma \frac{p}{1-p}\right)+p\left(y_{E, L}+\gamma\right) \\
& =E\left(y_{E}\right)=\bar{y}
\end{aligned}
$$

That is, the two technologies have the same expected return. It is also obvious that the new technology embodies lower risk since in both states the absolute difference between returns to the new technology and the mean return are less than for the existing technology. Thus, the existing technology is a mean-preserving spread of the new technology (variance is also easily calculated to verify this). This situation mirrors the potential case for skip-row corn.

It is a simple exercise to show that in the absence of insurance, since $U^{\prime}(x)>0$ and $U^{\prime \prime}(x)<0$, the new technology would always be adopted except in the trivial case of $p=0$, where the producer is indifferent. Of interest here is the expected utility maximizing technology choice in the presence of alternative insurance programs. Here, we depart from classic equilibrium analyses such as those contained in Rothschild and Stiglitz (1976), which analyze competitive market equilibrium outcomes, and instead focus on the expected utility maximizing insurance choices and technology adoption decisions under various government insurance program designs. The reason for this departure is that in the case of Federal Crop Insurance, the design and rating of the insurance offerings are a priori independent of the subsequent insurance purchases since the government must offer all insurances in the menu of contracts published, regardless of whether the offering of such contracts results in expected underwriting losses to the government. Thus the concepts of insurance market equilibrium take a less central role in the case of non-competitively offered government insurance. Also, insurance prices/rates are not set in equilibrium, but are rather predetermined by the government agency based on historical data. However, the government can determine how it chooses to use such data and how to structure the insurance market and products. Thus, in analyzing market outcomes here, it is sufficient to analyze the insured's insurance purchase and technology adoption decisions given alternative exogenously-determined government insurance program designs.

We consider two relevant cases. In the first case, it is assumed that both technologies are insurable, that insurance is offered at the actuarially fair cost of insurance of the existing technology, and that the insurance indemnifies in an amount equal to the actual loss below expected return. As further discussed below, this case mirrors that which exists for skiprow adoption under current RMA insurance rules, whereby both practices are pooled in the same insurance program. We show that adoption of the new technology would be completely crowded out in this case because of the design of the insurance market, and specifically, due to how insurance rates are determined by the government. The second case mirrors that which would exist under our proposed new rules for skip-row insurance.

Explicitly, in the low-yielding state, the indemnities paid under the existing and new technologies are $I_{E, L}=\bar{y}-y_{E, L}$ and $I_{N, L}=\bar{y}-y_{N, L^{\prime}}$ and in the highyielding state, indemnities are zero. Insurance is offered for both technologies at the actuarially fair insurance price of the existing technology, $r_{E}=p\left(\bar{y}-y_{E, L}\right)$. Employing a standard expected utility framework, we can write expected utility under each technology as $E U_{E}=p U\left(y_{E, L}-r_{E}+I_{E, L}\right)+(1-p) U\left(y_{E, H}-r_{E}\right)$, and $E U_{N}=p U\left(y_{N, L}-r_{E}+I_{N, L}\right)+(1-p) U\left(y_{N, H}-r_{E}\right)$. We can evaluate technology adoption decisions by evaluating whether $E U_{E}$ is greater than, equal to, or less than $E U_{N}$, or indeterminate. Substituting and eliminating terms, we have

$$
E U_{E}-E U_{N}=(1-p) U\left(J_{1}\right)-(1-p) U\left(J_{2}\right),
$$

where $J_{1}=\left(p-p^{2}\right) y_{E, L}+\left(p^{2}-p+1\right) y_{E, H}$

and

$J_{2}=-\left[\left(p^{3}-2 p^{2}+p\right) y_{E, L}+\left(-p^{3}+2 p^{2}-2 p+1\right) y_{E, H}-r p\right]$

$(p-1)$ 
Since $U^{\prime}(x)>0$, it is sufficient to show that $J_{1}-J_{2}>0$ to establish that $E U_{E}>E U_{N}$ (i.e. to establish that the existing technology will be adopted in lieu of the new technology under mispriced insurance). Straightforward but tedious algebra reveals that

$$
J_{1}-J_{2}=-\gamma \frac{p}{p-1}>0
$$

Thus, under such an insurance program design, adoption of the new technology would be completely crowded-out. ${ }^{1}$ The implication is that in the case of pooled insurance, all producers will find it advantageous to adopt the high risk technology. Here we derive the result assuming the insurance rate is equal to that of the actuarially fair rate for the high risk technology, but it is easily shown that this result is true regardless of the level of the rate, as long as the rate is identical for high and low risk, and feasible for the producer to purchase in the high risk case.

In the second case, it is assumed that both technologies are insurable, that insurance is offered for each technology at their respective actuarially fair costs (i.e. the insurance markets for each technology are separate), $r_{E}=p\left(\bar{y}-y_{E, L}\right)$ and $r_{N}=p\left(\bar{y}-y_{N, L}\right)$, and that the insurance again indemnifies in an amount equal to the loss below expected return. We can then write expected utility under each technology as

$$
E \tilde{U}_{E}=p U\left(y_{E, L}-r_{E}+I_{E, L}\right)+(1-p) U\left(y_{E, H}-r_{E}\right),
$$

and $E \tilde{U}_{N}=p U\left(y_{N, L}-r_{N}+I_{N, L}\right)+(1-p) U\left(y_{N, H}-r_{N}\right)$.

Again, we can evaluate technology adoption decisions by evaluating $E \tilde{U}_{E}-E \tilde{U}_{N}$. Subtracting and substituting, we have

$$
\begin{aligned}
E \tilde{U}_{E}-E \tilde{U}_{N}= & (1-p) U\left(J_{1}\right)-(1-p) U\left(J_{2}+\gamma p\right) \\
& -p U\left(J_{3}+\gamma p\right)+p U\left(J_{3}\right)
\end{aligned}
$$

where

$$
J_{3}=\left(2 p-p^{2}\right) y_{E, L}+\left(p^{2}-2 p+1\right) y_{E, H} .
$$

Since

and

$$
J_{1}-\left(J_{2}+r p\right)=-\left(r p^{2}\right) /(p-1)>0,
$$

$$
\begin{aligned}
\left(J_{2}+r p\right)-J_{3}> & \lim _{r \rightarrow \frac{y_{E, H}-y_{E, L}}{1+p /(1-p)}}\left[\left(J_{2}+r p\right)-J_{3}\right] \\
= & \left(p-p^{2}\right) y_{E, H}-\left(p-p^{2}\right) y_{E, L}>r p>0,
\end{aligned}
$$

it follows that $J_{1}>J_{2}+r p>J_{3}+r p>J_{3}$, and that the relationship between $E \tilde{U}_{E}$ and $E \tilde{U}_{N}$ is indeterminate on this basis. However, we can rely on the assumption that $U^{\prime \prime}(x)<0$ to argue that the new technology is strictly preferred under this insurance regime by simply showing that the insured net returns under the existing technology are a mean-preserving spread of the returns under the new technology. Clearly, the expected insured net returns for both technologies are equal since the insurance is priced actuarially fair. Thus, all that is required to prove a mean preserving spread is to show that the net insured return to the new technology in the low-yielding state, $\tilde{R}_{N, L^{\prime}}$ is greater than the net insured return to the existing technology in the low-yielding state, $\tilde{R}_{E, L^{\prime}}$ and vice-versa in the high-yielding state. Straightforward calculations show that $\tilde{R}_{N, L}-\tilde{R}_{E, L}=\gamma p>0$, and $\tilde{R}_{E, H}-\tilde{R}_{N, H}=r p^{2} /(p-1)>0$. Thus, net insured returns for the new technology second order stochastic dominate insured returns under the existing technology when insurance is priced fairly, and thus a riskaverse expected utility maximizing producer will adopt the new technology.

If we allow for relative insurance subsidies (which favor the higher risk technology), allow for differences in expected yields and distributional shape, and/or allow for mispriced insurance across producers, then the results of the theoretical results presented above are indeterminate and will depend on the specific utility function, level of risk aversion, levels of subsidization, and the resulting yield distributions. Thus, whether the skip-row technology case in our application would sufficiently conform to the theoretical results above is ultimately an empirical question that we investigate below. First, however, we provide additional context about the insurance rules for skip-row corn currently in use by RMA and the expected yield distribution impacts.

\section{Skip Row Insurance Program Background}

Prior to the 2008 crop year, RMA did not offer skip-row corn insurance to producers via the regular insurance program, but only under restrictive written agreements. Starting in 2008, RMA began offering insurance for skip-row corn in selected counties in Kansas, Colorado, and Nebraska without a written agreement, subject to specific requirements. Under the 2008 rules, RMA required producers to insure their skiprow crops separately from conventionally-planted crops, and to maintain separate yield databases. In

1. It is well-accepted that government insurance programs should seek to replicate what would happen in an unfettered and/or competitive market in the absence of intervention. Thus, we say that the adoption of the new technology is "crowded-out" in this case since it is the existence of the mispriced insurance that is precluding adoption. 
addition, RMA used the Farm Service Agency (FSA) percentage acreage factors to determine the number of acres planted to the crop for insurance purposes. The FSA percentage acreage factors were intended to determine planted acreage for fields using skip row patterns by taking into account the proportion of skipped rows; they work simply by multiplying the number of actual acres planted by the factor in order to obtain the adjusted planted acreage. For example, if 100 acres of land were skip-row planted, it would be recorded and treated as having $100 \times 0.5$ $=50$ acres planted. In terms of calculating yield (or production per acre), if 10,000 bushels of corn were actually produced on those 100 acres of land, the factored yield would be calculated as $10,000 \div(100 \times$ $0.5)=200 \mathrm{bu}$./acre, whereas the conventional calculation would have been simply $10,000 \div 100=100$ bu./ acre. In addition, new skip-row producers' purchasing insurance for allowable skip-row patterns under the 2008 rules were required to use Transitional Yields (T-Yields) to establish their initial insurance guarantee (known as Actual Production History, or APH). However, the T-Yields were not factored into the APH determination. This resulted in producers effectively receiving coverage for half of the T-Yield $\mathrm{APH}$, and at approximately half of the effective premium rate. Except under very unrealistic distribution assumptions, the fair premium associated with half of any given level of coverage will typically be much lower than half of the premium associated with that level of coverage.

At the time, there was concern that the use of these factors unfairly penalized producers adopting skiprow technologies since the insurance guarantees were reduced, and that this was disincentivizing producers from adopting skip-row cropping (Palic et al. 2008). From an economic perspective, this is troubling since the unfair provision of insurance across practices/ technologies may have been crowding out the adoption of skip-row technology.

Starting in 2009, RMA announced new rules for skip-row corn in the region. In addition to expanding skip-row coverage to more counties, RMA announced that the FSA-planted acreage factors would no longer be employed, and that skip-row and conventional planted crops would be pooled together as a single insurable practice. Still, concerns remain that skip-row and conventional practices may not embody the same risk profile and production capabilities. From an economic and actuarial standpoint, combining significantly dissimilar risks into the same insurance product opens the door for mispricing risks, adverse selection, and other market distortions
Table 1. Summary of RMA Skip-Row Corn Insurance Rules

\begin{tabular}{llll}
\hline & Coverage & $\begin{array}{l}\text { Premium Rate } \\
\text { Determination }\end{array}$ & $\begin{array}{l}\text { FSA } \\
\text { Factors }\end{array}$ \\
\hline $\begin{array}{lll}\text { Pre-2008 Rules } \\
2008 \text { Rules }\end{array}$ & Neparate & Pooled & N/A \\
$\begin{array}{l}\text { 2009/Current } \\
\text { Rules }\end{array}$ & Pooled & Pooled & Yes \\
$\begin{array}{c}\text { Proposed } \\
\text { Rules }\end{array}$ & Separate & Separate & No \\
\hline
\end{tabular}

This table provides a summary of RMA rules for skip-row corn in eligible counties in the Central Great Plains. "Coverage" refers to whether skip-row and conventional practices are insured under the same/pooled or separate policies. "Premium Rate Determination" refers to whether the premium rate for the insured crop is the same/pooled for both skiprow and conventional practices, or if rates are maintained and determined separately by practice. "FSA Factors" refers to whether or not FSA factors are used to determine skip-row acreage for yield and rate determination purposes.

if the inappropriate provision of the insurance alters otherwise optimal behaviors. These issues are important from an economic perspective as, generally, government insurance programs should seek to replicate how an unfettered market would perform in similar situations, and thus should avoid crowding out innovations which otherwise would be adopted in the absence of such programs unless explicitly intended (Jaffee 2006; Woodard et al. 2011). If the production characteristics among practices are significantly different-and if feasible from an operational perspective-then treating skip-row and conventionallyplanted practices as separate insurable practices may be warranted. Thus, we propose that RMA should consider maintaining coverage and rates for skip-row separately from conventional practices/technologies, and should not employ the FSA factors since they artificially lower coverage in some instances and add unnecessary complications in others. A summary of past and current RMA rules and our proposed rule are provided in Table 1.

\section{Production Impacts of Skip-Row Technology and Implications for Insurance}

As noted, there is a potential for skip-row corn to outperform conventionally-planted corn when moisture conditions are less than optimal. Likewise, conventionally-planted crops may tend to have higher yields when weather conditions are favorable. This 
suggests two potential impacts due to skip-row adoption that are relevant for insurance: impacts on average production capabilities (i.e. the mean, or expected yield), and impacts on yield risk (i.e. insurance costs, variance, and other higher order moments). Accordingly, the adoption of skip-row will have two primary impacts on its actuarial profile versus conventional practices. The first is the impact on the APH, the measure used to determine insurance guarantees. $\mathrm{APH}$ equals the average of the last four to ten years of farm-unit data. The second impact is on the expected insurance losses.

Recall that under the current 2009 RMA rules, skip-row and conventional practices are insured together and assigned a common APH and insurance rate. Whether the expected changes in risk and expected yields from using skip-row patterns relative to conventional patterns are such that they justify the use of the same APH guarantee and rating structure (i.e. schedule of insurance rates) is an empirical question. Because skip-row corn may have higher or lower expected yields on average, if the APH is established for conventionally-planted corn but applied to skip-row insured corn, the APH may be biased up or down. This may result in artificially high (or low) insured APH's for skip-row, and subsequently may impact indemnities under any particular loss event. Second, even independent of skip-row impacts on APH, skip-row cropping may also embody a lower risk of insured loss. Thus, skip-row may have lower expected insurance losses relative to conventional losses for any given level of equivalent bushel/ acre coverage. If not properly accounted for, these insurance coverage and rate effects have the potential to impact technology adoption and lead to adverse planting incentives.

To provide context, Figure 1 presents agronomic trial data from Lyon et al. (2009) of matched skiprow and conventionally-planted corn yields for selected counties in the Central Great Plains. The data

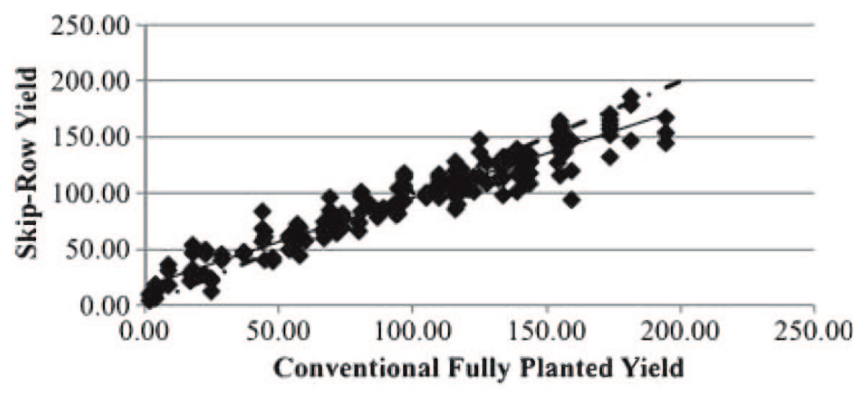

Figure 1. Corn Yield (bu./acre) Trial Data, Skip-Row vs. Conventional (Lyon et al. 2009)
Table 2. Summary Statistics-Agronomic Trial Data

\begin{tabular}{lrr}
\hline & Conventional & Skip-Row \\
\hline Mean & 95.427 & 92.530 \\
Standard Deviation & 47.979 & 39.770 \\
Semi-Variance & 1197.244 & 823.063 \\
1st Percentile Yield & 2.090 & 10.047 \\
5th Percentile Yield & 17.047 & 21.986 \\
10th Percentile Yield & 23.149 & 40.084 \\
Median & 97.024 & 96.069 \\
90th Percentile Yield & 155.876 & 145.839 \\
95th Percentile Yield & 173.656 & 154.968 \\
99th Percentile Yield & 194.240 & 170.438 \\
\hline
\end{tabular}

This table presents summary statistics for skip-row and conventional matched yields $(N=270)$ from Lyon et al. (2009). The correlation between skip-row and conventionally-planted yields was 0.954 .

were collected from 2004-2006 from 23 trials conducted in Nebraska, Kansas and Colorado; several measures were taken to ensure that the data are extensible over the region. ${ }^{2}$ In Figure 1, the conventionally-planted yield outcome (bu./acre) is plotted on the $x$-axis, while the corresponding skip-row yield is on the $y$-axis ( $N=270$ observations). The dashed line is a $45^{\circ}$ line emanating from the origin. The solid line is a trend line from an OLS regression. We do not argue that a linear OLS is the best specification, but include it for expositional purposes. Figure 1 indicates that, on average, skip-row planting tends to outperform conventionally-planted when conventionally-planted yields are less than approximately $80-90 \mathrm{bu}$./acre, and vice-versa when yields are above $80-90 \mathrm{bu}$./acre. This is indicated by the intersection point of the dashed line and the solid trend line. Summary statistics for the data are presented in Table 2. Skip-row production has lower yield variability (according to variance, semi-variance, and distances between quantiles), and outperforms conventional planting at quantiles below the mean, and vice-versa above the mean.

\section{Empirical Analysis}

The empirical analysis seeks to address three primary questions. First, which practice/technology (skip-row or conventional) would be preferred in the absence of insurance? Second, did the 2008 RMA insurance rules disincentivize the adoption of skiprow production? And third, are the practices suitably

2. We refer the interested reader to Lyon et al. (2009) for more in-depth details on the trial data. 
similar to justify being combined as a single insurable practice as under the current rules, or should they be maintained as separate insurable practices? To address these questions, first a crop yield simulation model of skip-row and conventional yields is developed. Next, an expected utility analysis is conducted to evaluate insurance and technology adoption choices.

\section{Skip-Row Crop Yield Simulation Model}

Empirical assessment of skip-row risk presents a special challenge since the practice has only recently been introduced to the region, and skip-row performance has not yet been observed over a large number of years in any single location. However, reliable trial data do exist, which contain side-by-side comparisons of skip-row and conventionally-planted yields over a wide range of weather outcomes and regions. We develop a simulation to derive credible skip-row distributions by combining information from agronomic side-by-side trial data with a larger farm-unit level dataset. By combining information from the two datasets, more efficient estimates of the skip-row distribution can be obtained. The trial data are from Lyon et al. (2009) and span from 2004$2006(N=270)$. The larger dataset of conventional yields is obtained from the RMA Type 15 producer yield records, and consists of 130,080 conventional corn yield observations from 1996-2008. The Type 15 database is the RMA database where producer-level historical yields are maintained; they were provided by RMA and deemed appropriate for use in this application. Unless otherwise noted, usage of the Type 15 yields are assumed to refer to detrended yields in order to accurately account for technology gains through time. The yield detrending process is detailed in the next section.

The simulation methodology employs a multivariate nonparametric simulation technique to derive augmented skip-row yield distributions, insurance rates, and to perform expected utility analyses. The methodology involves several steps. First, a nonparametric bivariate density of the difference between skip-row and conventional yields is constructed using the yield trial data to establish the relationship between skip-row and conventional yields. We augment the trial data with the larger database of conventional yields by employing a resampling procedure, whereby a conventionally-planted yield is first sampled (based on models calibrated with the Type 15 data), and then a skip-row yield is constructed by simulating a skip-row difference yield from the estimated bivariate density, conditional on the level of the conventional yield sampled from the Type 15 data. ${ }^{3}$ Explicitly, the procedure is as follows. First, a variable is constructed for the difference between skip-row and conventionally-planted yields using trial data as $x_{i}=y_{i}^{S}-y_{i}^{F} \forall i \in\{1,2, \ldots N\}$, where $i$ is the observation index, and $y_{i}^{S}$ and $y_{i}^{F}$ are paired skip-row and convention yield observations from the trial data. Alternatively, in matrix notation we have

$$
\mathbf{x}_{N \times 1}=\mathbf{y}_{N \times 1}^{S}-\mathbf{y}_{N \times 1}^{F},
$$

where for exposition we include the dimensions of the matrices. Next, a bivariate density is constructed of $x=y^{S}-y^{F}$ and $y^{F}, f\left(x, y^{F}\right)$. Using this density, we then construct the conditional density of $f\left(x \mid y^{F}\right)$. With this density in hand, conventional yield draws, $\bar{y}^{T 15}$, are simulated from the empirical detrended yield density from the Type 15 yield dataset, $\hat{f}\left(y^{T 15}\right)$. Skiprow differences, $\bar{x}$, are then simulated from $\hat{f}\left(x \mid \bar{y}^{T 15}\right)$ conditional on $\bar{y}^{T 15}$. Unit-level simulated skip-row yields are then calculated as $\bar{y}^{S}=\bar{y}^{T 15}+\bar{x}$.

We estimate the joint density of $x$ and $y^{F}, f\left(x, y^{F}\right)$ as a bivariate Gaussian kernel density using a product kernel and bandwidth, $\mathbf{h}_{1 \times 2}$, as suggested by Bowman and Azzalini (1997, p. 31). ${ }^{4}$ Letting

$$
\mathbf{Z}_{N \times 2}=\left[\mathbf{x}_{N \times 1}, \mathbf{y}_{N \times 1}^{F}\right],
$$

we can calculate the bandwidth as

$\mathbf{h}_{1 \times 2}=\operatorname{MED}[|\mathbf{Z}-\operatorname{REP}[M E D[\mathbf{Z}], N, 1]|] \cdot\left(0.6745 \times N^{1 / 6}\right)^{-1}$,

where the matrix function $R E P[\mathbf{Z}, N, M]$ is an $N \times M$ tiling of matrix $\mathbf{Z}$, and the function $M E D[\mathbf{Z}]$ results in an $N \times 1$ vector with $m$ th element equal to the median of the $m$ th column of $\mathbf{Z}$. Next, the bivariate Gaussian kernel density is estimated as

3. The primary advantage of our simulation approach is that it is not necessary that the marginal distribution of conventional yields from the trial data be a "good" estimate of the underlying "true" marginal distribution, only that it suitably spans the support of the "true" marginal distribution, and that the data are extensible across the region as it regards impacts from management practices, soil characteristics, etc. From a statistical point of view, the only relevant information contained in the trial data is that which pertains to the relationship between skip-row and conventional yields, conditional on the conventional yield outcome. Indeed, the marginal distributions derived from the trial data are otherwise not relevant, nor is it required that they be accurate depictions of the "true" underlying marginals in order to effectively implement our procedure. In practice though, care should be taken to ensure that the data generated in the agronomic trials are representative of yields in the region.

4. We also investigated using a shaped multivariate normal kernel with bandwidth $h=(4 / 5 N)^{1 / 6}$ in lieu of the product kernel as outlined in Simar and Wilson (1999), but found little practical difference in the results. 
$\hat{f}\left(\mathbf{z}_{1 \times 2}\right)=N^{-1} \mathbf{h} \boldsymbol{\imath}^{-1} \sum_{i=1}^{N} K\left(\left(\mathbf{z}-\mathbf{Z}_{i}\right) \cdot / \mathbf{h}\right)$,

where $\quad \imath=[1,1]^{\prime}$,

$\mathbf{Z}_{i}$ is the $i$ th row of the original data, $\mathbf{Z}=\left[\mathbf{x}, \mathbf{y}^{\mathbf{F}}\right]$,

$K\left(\left[t_{1}, t_{2}\right]\right)=\frac{e^{\frac{-t_{1}^{2}}{2}}}{\sqrt{2 \pi}} \cdot \frac{e^{\frac{-t_{2}^{2}}{2}}}{\sqrt{2 \pi}}$

and "./" denotes element-by-element matrix division. The marginal density of $y^{F}$ is obtained by integrating out $x$ as

$\hat{f}\left(y^{F}\right)=\int_{-\infty}^{\infty} \hat{f}(\mathbf{z}) d x=\int_{-\infty}^{\infty} \hat{f}\left(\left[y^{F}, x\right]\right) d x$.

Estimates of the conditional density of $x$ conditional on $y^{F}, \hat{f}\left(x \mid y^{F}\right)$ can then be constructed using the definition of a conditional density. Note that this density is used to simulate skip-row difference variables, $\bar{x}$, conditional on the simulated conventionally-planted variates, $\bar{y}^{F}$, whereby the simulated conventionallyplanted conditioning yield will be drawn from the empirical yield distribution from the larger dataset, $\bar{y}$ ${ }^{T 15}$ (in our case, the Type 15 data). The simulated skiprow yield is then calculated as the sum of the simulated conventionally-planted conditioning yield and the simulated skip-row difference yield to obtain $\bar{y}^{S}=\bar{y}^{T 15}+\bar{x}$. Since the skip-row simulated yield is bounded below at zero, we must truncate our conditional distribution of $\bar{x}$ from below at $-\bar{y}^{F}$ to ensure that $\bar{y}^{S}$ is always greater than zero. Put simply, this avoids drawing negative yields. This is accomplished by employing the reflection method of Silverman as illustrated in Simar and Wilson (1999). The conditional distribution of $x$ on $y^{F}$ is itself a univariate distribution, thus a reflection point is needed at $-\bar{y}^{F}$ for $x$. The reflection method in this case with only a lower bound is implemented by assigning a zero value to $\hat{f}\left(x \mid \bar{y}^{F}\right)$ when $x$ is less than $-\bar{y}^{F}$; and, when $x$ is greater than - $\bar{y}^{F}$, the density of $\hat{f}\left(x \mid \bar{y}^{F}\right)$ is equated to the untruncated density evaluated at $x$, plus the un-truncated density value evaluated at the reflected value of $x$ around $-y^{F}$, or $x_{r}=-\bar{y}^{F}-\left(x-\left(-\bar{y}^{F}\right)\right.$. Thus we have

$$
f\left(x \mid \bar{y}^{F}\right)=\left\{\begin{array}{lr}
0 & \text { if } x<y^{F}, \\
f\left(y^{F}\right)^{-1} N^{-1} \mathbf{h} i^{-1}\left(\sum_{i=1}^{N} K\left(\left(\mathbf{z}-\mathbf{Z}_{i}\right) . / \mathbf{h}\right)\right. \\
\left.\quad+\sum_{i=1}^{N} K\left(\left(\mathbf{z}_{r}-\mathbf{Z}_{i}\right) \cdot / \mathbf{h}\right)\right) & \text { otherwise }
\end{array}\right.
$$

where $\mathbf{z}_{r}=\left[x_{r}, y^{F}\right]$. The empirical distribution for drawing from the Type 15 data is constructed using the detrended yields as described below. We simulate 500 draws from the empirical distributions for each unit, and then simulate 500 draws from the conditional difference distribution in order to calculate simulated skip-row yields for each practice for each of the farm units in the sample. ${ }^{5}, 6$ Insurance loss costs, expected yields, risk measures, and expected utility for each practice are then calculated using the simulated draws.

\section{Constructing Forward-looking Empirical Distributions of Conventional Yields}

To construct forward-looking conventional yield distribution estimates from the Type 15 data for use in the crop yield simulation model above, yields from the Type 15 data are first detrended to account for technological advances through time. The resulting yields deviations from trends are then used to construct empirical farm-unit level yield distributions. The fact that the typical farm-unit yield series in these data are short (10 years or less)-coupled with the frequent presence of total yield losses in the Central Great Plains region-render farm-unit level OLS trend estimation unattractive. The typical approach to increasing the estimation efficiency in this case is to pool units together and then estimate trends for some region or group of similar units. This allows for a reduction in the impact of sampling variability on estimated trends (Atwood, Shaik, and Watts 2003). One typical manner in which this is implemented is to estimate county-level trends and then apply the estimated county trend to detrend the farm-level unit yields. This $\mathrm{ca}^{\wedge} \mathrm{n}$ be justified with the reasoning that farms within a county are typically subject to similar technology trends and climactic conditions, and thus pooling can increase estimation efficiency with little risk of inducing bias. Thus, we utilize county-level yields when detrending. Specifically, county level yields from the National Agricultural Statistics Service (NASS) are used to estimate robust trends using data from 1972-2008. The basic concepts underlying this approach are well-established (see e.g. Atwood, Shaik, and Watts 2003; Woodard, Sherrick, and Schnitkey 2011).

5. We assessed the impact of using a sample size of 5,000 versus 500 for one of the coverage level/plant configurations combinations. The difference at the farm-unit level was negligible, and the difference for the county level summaries of the 40,336 farm-unit level simulations was essentially zero. This was not surprising, given that Latin Hypercube sampling was employed.

6. The Mersenne-Twister algorithm is employed to generate the uniform variates needed for the inverse transform sampling. Latin Hypercube sampling is employed to increase simulation sampling efficiency. Descriptions of these well-established methods can be found in most elementary simulation textbooks. 
A feature of county-level yields in this region is that there is extreme volatility in yields from year-toyear, and the occurrence of near-total loss years can potentially create outlier problems. To address this issue, we employ a robust Iterative Reweighted Least Squares Huber Estimator when estimating the trend. With the trend estimate in hand, we can then obtain detrended unit-level yields as

$$
y_{t}^{T 15}=y_{t}^{u T 15} \times \frac{Y_{2009}^{T r}}{Y_{t}^{T r}}
$$

where $Y_{t}^{T r}=\hat{\beta}_{1}+\hat{\beta}_{2} t$ is the county trend yield in year $t$, $y_{t}^{u T 15}$ is the trended farm-unit level conventional yield from the Type-15 data, $Y_{t}^{T r}$ is the fitted county yield from the regression of time on NASS yields (i.e. the "trend yield"), $t$ is the year, and $\beta^{\prime}$ s are coefficients to be estimated from the regression of time on NASS county yields. In our case, the Type 15 data contain historical yields from 1996-2008 at the farm-unit level. Thus, to construct estimates of the 2009 conventional yield distributions, yields are detrended to 2009. Note that this detrending method implicitly assumes constant relative yield risk growth through time. As Woodard (2008) and Woodard, Sherrick, and Schnitkey (2011) point out, this assumption is consistent with RMA's loss cost system requirements and assumptions. The high frequency of total yield loss events in this region suggests that this assumption is likely reasonable for this application. Assuming other forms of yield risk or yield trend form would impact the nominal estimated rate and loss levels for skip-row and conventionally-planted production, but should not change the results concerning the relative relationships between skip-row and conventional yields significantly.

\section{Estimation of Insurance Loss Costs}

Since adopting skip-row practices has a primary impact on the yield loss component, we restrict attention to the traditional APH yield insurance product in the insurance analysis. As noted, we simulate yield observations of $\bar{y}^{T 15}$ and $\bar{x}$ from $\hat{f}\left(y^{T 15}\right)$ and $\hat{f}\left(x \mid \bar{y}^{T 15}\right)$, then calculate simulated skip-row yields conditional on $\bar{y}^{T 15}$ as $\bar{y}^{S}=\bar{y}^{T 15}+\bar{x}$. Thus, we can calculate loss cost in a particular case for the standard yield insurance, as well as expected loss costs as,

$$
L C=\frac{\operatorname{Max}(0, A P H \times \text { Cover }-\tilde{y})}{(A P H \times \text { Cover })}
$$

and

$$
E(L C)=\frac{\sum_{i=1}^{I} \operatorname{Max}\left(0, A P H \times \operatorname{Cover}-y_{i}\right)}{A P H \times \text { Cover } \times I}
$$

where $I$ is the number of yields available (or simulated), $A P H$ is the unit APH, $y_{i}$ is the simulated yield outcome (skip-row or conventional), and Cover is the (\%) coverage level.

\section{Expected Utility Analysis}

To investigate the impacts of various insurance program designs on skip-row adoption in an economic framework, we conduct an expected utility analysis using the simulated yields obtained from the crop simulation model described above. Since production costs for skip-row and conventional are typically similar, we focus on the expected utility of crop revenues net of insurance costs (price normalized). Actuarially fair insurance costs are estimated from the simulation model for skip-row and conventional planting practices, where the indemnity function for the yield-based insurance is defined above. ${ }^{7}$ The analysis adjusts farmer-paid premiums using the current subsidy structure as published by RMA. Following Holt and Laury (2002), we adopt a power utility function. This utility function exhibits constant relative risk aversion (CRRA) and can be expressed as $U(x)=x^{1-r} /(1-r)$, where $x$ is the relevant return (revenue plus any insurance indemnity) net of insurance costs, and $r$ is the risk aversion coefficient. Expected utility can then be calculated as

$$
E U=\int_{0}^{\infty} U(x) d G(x)
$$

where $G(x)$ is the distribution of returns from the simulation model. Results are presented at the $85 \%$ coverage level for a representative producer in Thomas, Kansas at various levels of risk aversion as characterized by Holt and Laury (2002).

Expected utility is evaluated over four different scenarios for both conventional and skip-row practices: (1) no insurance, (2) insurance under 2008 rules, (3) insurance under 2009/current rules, and (4) insurance under our proposed rules. Recall that under 2008 RMA rules, skip-row producers without es-

7. While revenue insurance is also popular in this region, we focus the analysis on a yield-based insurance product in order to maintain focus on the component impacted by skip-row adoption (i.e. yields). The results likely hold similarly for revenue products, however, since skip-row and conventional yields are highly correlated. 


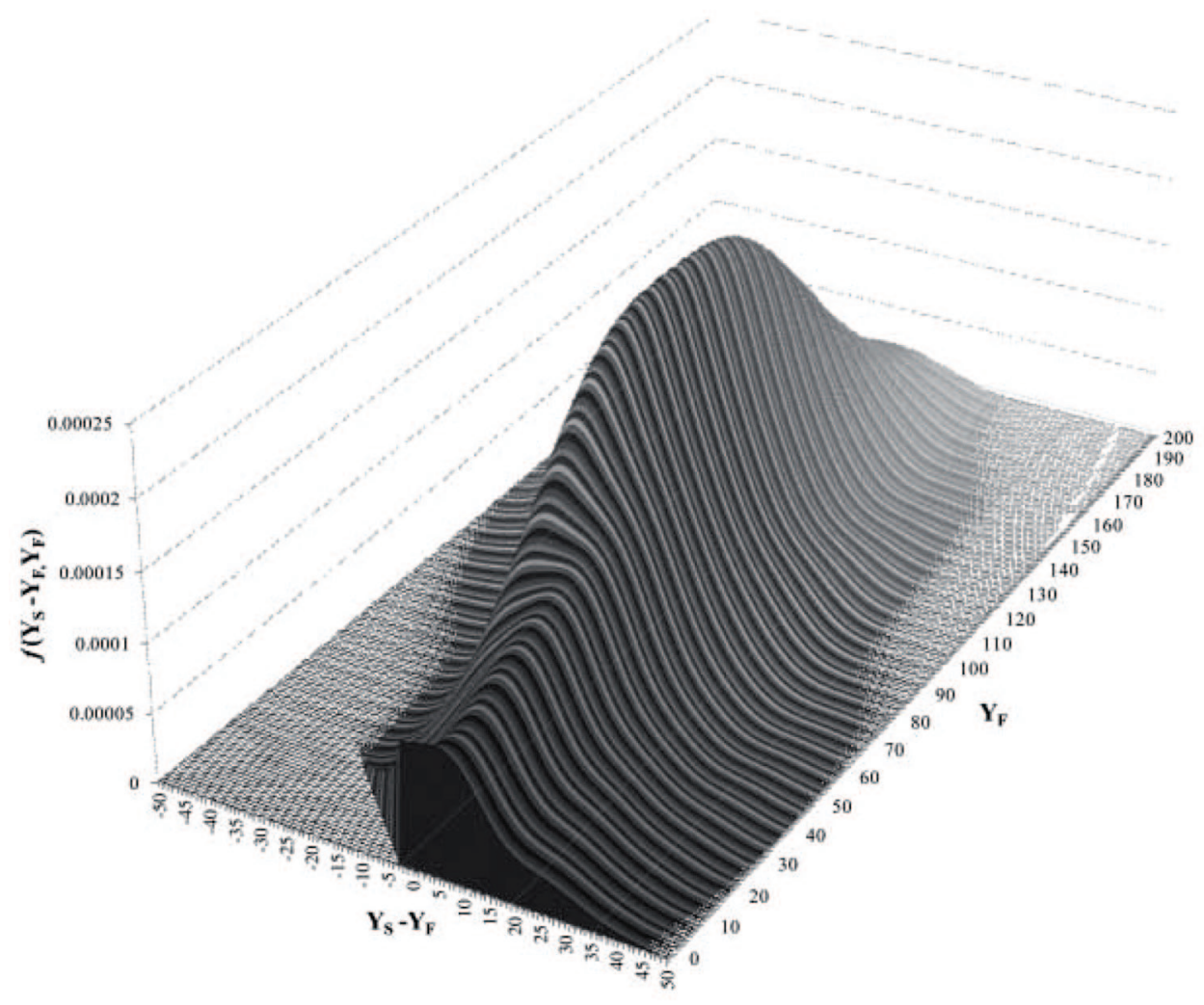

Figure 2. Joint Distribution of $x=y^{S}-y^{F}$ and $y^{F}(N=270)$

tablished databases received effectively one-half of the T-yield APH for insurance purposes, and the insurance cost was also one-half the conventional premium. ${ }^{8}$ Under the current 2009 RMA rules, skip-row is combined with conventional production for insurance purposes, and also is charged the same rate. Thus, new skip-row producers will receive an APH equal to their conventional $\mathrm{APH}$, and also receive the established conventional yield premium rate. Based on previous research about skip-row yields in this region-which suggests that skip-row in this region has a higher expected yield and lower risk/expected loss costs than conventional-we propose an alternative set of rules which would establish skip-row and conventional as separate practices with separate rates and APH databases. In the analysis, the insurance premium rate and $\mathrm{APH}^{\prime}$ s are determined by the expected loss cost (minus subsidy) and the expected yield from the simulated yields, respectively.

\section{Results - Distribution and Expected Loss Cost Impacts of Skip-Row Technologies}

Figure 2 presents the truncated joint distribution of $x$ and $y^{F}$ estimated from the trial data. As ev- idenced by Figures 1 and 2, the data cover a large spectrum of possible outcomes. In general, the difference between skip-row and conventional production increases as the conventionally-planted yield increases, which is consistent with previous research. However, there is still some variability in the relationship between skip-row and conventional production over different events. Thus, in regions with high frequencies of yields below $80-90 \mathrm{bu}$./acre, more mass will be contained further away from zero for skip-row relative to conventional cropping. As noted by Lyon et al. (2009), the expected value of skip-row yields exceeds that of conventionallyplanted when conventionally-planted yields are less than approximately $80-90 \mathrm{bu}$./acre. This does not imply that skip-row yields will always exceed those of conventionally-planted yields when conventionally-planted yields are below $80 \mathrm{bu}$./acre, but only on average. The nature of the bivariate joint density explicitly takes this into account.

To illustrate the impact of skip-row in terms of the simulated augmented distribution, Figure 3 presents kernel distributions from the simulated values for skip-row and conventional yields for Thomas County, Kansas, a high production county. Figure 3 


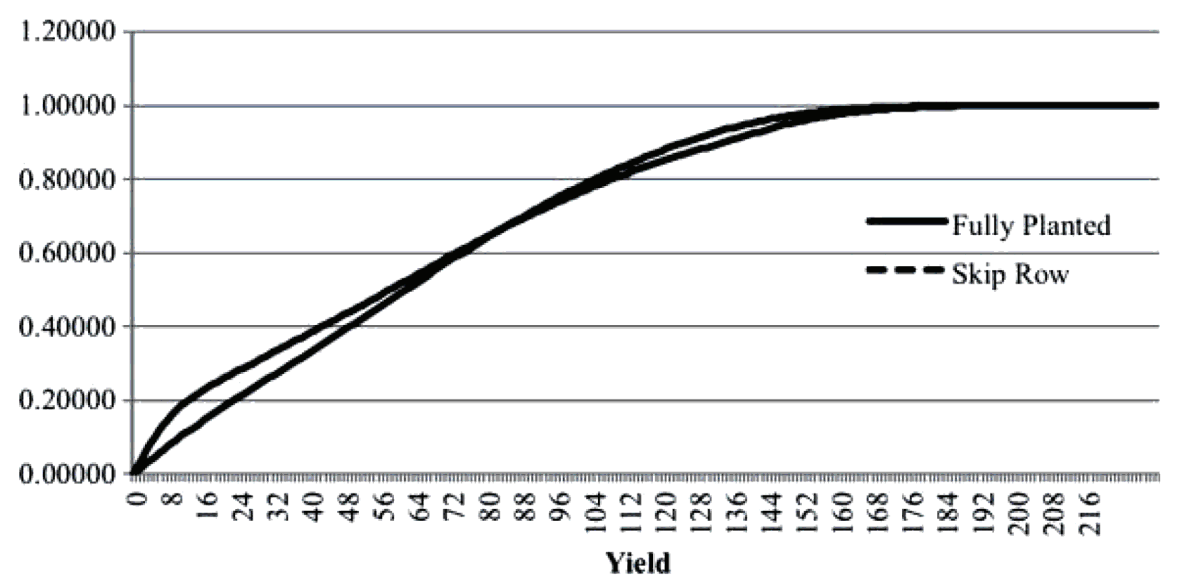

Figure 3. Simulated Augmented Yield CDF's, Thomas KS
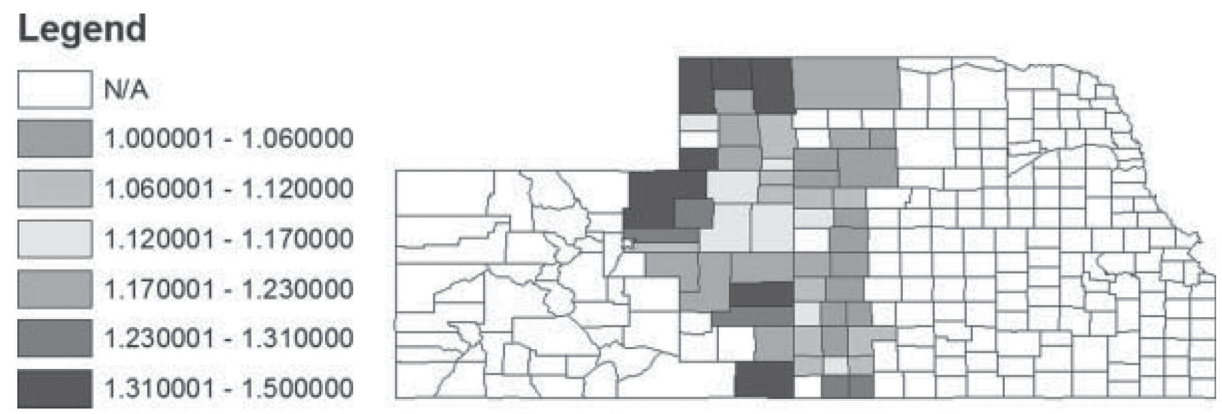

Figure 4. Ratio of Expected Yield, Skip-Row to Conventional

illustrates that density is redistributed closer to the mean for skip-row, and that skip-row also has less density far out in the tails. This is a result of the fact that there is a high frequency of total loss (or neartotal loss) events for conventional yields in this county. Also, the conventionally-planted distributions have more mass at higher yield levels, an effect attributable to the fact that conventionally-planted yields tend to outperform skip-row when conventionally-planted yields are above $80-90 \mathrm{bu}$./acre. Figure 4 presents a map of the ratio of skip-row and regular planted expected yields. As the Figure illustrates, in the eastern region, skip-row and conventional production performed similarly in terms of expected yield (with skip-row modestly outperforming); in the western region, skip-row tended to significantly outperform (30\% to $50 \%$ higher expected yield in some counties). This result is somewhat expected since this region tends to be more drought-prone, and has lower expected yields (map not presented).

To assess the impact of skip-row patterns on expected insurance losses, the average simulated insurance loss costs (expressed as a percentage of the liability, or bu./acre coverage) are estimated using the simulation procedure described. ${ }^{9}$ Table 3 reports results for the expected loss cost analysis for skip-row and conventionally-planted yields at different coverage levels, aggregated over all units in the region. ${ }^{10}$ The first block column of results in Table 3 report conventionally-planted expected loss costs at each coverage level; the second block column reports skiprow loss costs, and contains three individual sub-columns, one for each of the three methods for determining skip-row APH: (1) T-Yield APH (reflecting 2008 rules), (2) Conventional Planted Expected yield (reflecting 2009 and current rules), and (3) Skip-Row Expected Yield (reflecting proposed rules).

9. The county expected unit loss costs generated from the simulations should not be construed as final rates for FCIC premium determination purposes. In practice, RMA makes several adjustments to base loss costs/rates, and also implements specific weightings both across units and across years. We do not replicate all of those steps, nor is doing so necessary to evaluate the impacts of skip-row which are of interest here.

10. Our results were consistent across all seed densities and skip-row planting configurations regarding rankings relative to conventionallyplanted practices (results not reported). Please see Lyon et al. (2009) for a discussion of planting configurations and seed densities used in the trials. 
Table 3. Simulated Expected Loss Costs - Average of All Units/Counties

\begin{tabular}{|c|c|c|c|c|c|}
\hline \multicolumn{2}{|c|}{ Practice $\rightarrow$} & \multirow{2}{*}{$\begin{array}{c}\text { Conventional } \\
\text { Conventionally- } \\
\text { Planted Expected } \\
\text { Yield }\end{array}$} & \multicolumn{3}{|l|}{ Skip-Row } \\
\hline Det & $\begin{array}{c}\mathrm{APH} \\
\text { etermination } \\
\rightarrow\end{array}$ & & $\begin{array}{c}\text { T-Yield } \\
\text { (2008 Rules) }\end{array}$ & $\begin{array}{l}\text { Conventionally- } \\
\text { Planted Expected } \\
\text { Yield (Current Rules) }\end{array}$ & $\begin{array}{c}\text { Skip-Row } \\
\text { Expected Yield } \\
\text { (Proposed Rules) }\end{array}$ \\
\hline Coverage & $85 \%$ & $30.20 \%$ & $11.35 \%$ & $19.65 \%$ & $23.23 \%$ \\
\hline \multirow[t]{7}{*}{ Level } & $80 \%$ & $29.18 \%$ & $10.65 \%$ & $18.43 \%$ & $21.86 \%$ \\
\hline & $75 \%$ & $28.17 \%$ & $9.95 \%$ & $17.22 \%$ & $20.49 \%$ \\
\hline & $70 \%$ & $27.19 \%$ & $9.24 \%$ & $16.03 \%$ & $19.13 \%$ \\
\hline & $65 \%$ & $26.20 \%$ & $8.54 \%$ & $14.85 \%$ & $17.78 \%$ \\
\hline & $60 \%$ & $25.22 \%$ & $7.82 \%$ & $13.67 \%$ & $16.42 \%$ \\
\hline & $55 \%$ & $24.25 \%$ & $7.11 \%$ & $12.49 \%$ & $15.05 \%$ \\
\hline & $50 \%$ & $23.28 \%$ & $6.40 \%$ & $11.31 \%$ & $13.68 \%$ \\
\hline
\end{tabular}

In general, loss costs are substantially lower for skip-row production. The corresponding conventionally-planted yield expected loss cost was typically about $50 \%$ to $100 \%$ in excess of that for skip-row. For example, the skip-row rate (under the conventional $\mathrm{APH}$, sub-column 2) at $85 \%$ (50\%) coverage is approximately $19.65 \%(11.31 \%)$, versus a rate of $30.20 \%(23.28 \%)$ for conventional production. Skiprow rates (i.e. loss costs) were slightly higher for the skip-row adjusted APH (sub-column 3), versus under the conventional APH (sub-column 2), reflecting the fact that the skip-row expected yield was higher than the conventionally-planted, on average, in the sample. However, it was still much less than the loss cost for conventional practices. This finding suggests that even if skip-row APH's are allowed to adjust upward over time in a combined practice insurance program (as under the current rules), producers adopting skip-row cropping will still receive rates that are too high since they are lower than conventional yield loss costs. Recall that under the 2008 rules, new skip-row producers effectively received a fraction of the quoted T-Yield as their yield guarantee (approximately $50 \%$ ) because of the manner in which FSA factors were used to determine acreage. To illustrate what the expected impacts were from imposing this deflated T-Yield as the APH for skip-row under the 2008 rules, the first sub-column under skip-row presents expected loss costs using the T-Yield and FSA planted acreage factors when calculating indemnities.

The results of our study imply that rates for skiprow are massively overpriced relative to conventional practices at all given levels of coverage. This is somewhat expected, though since under the 2008 rules, a skip-row producer effectively received a fraction of the coverage that would have been provided for conventionally-planted (as determined by the FSA factor) at a fraction of the rate. However, generally there is not a one-to-one proportional tradeoff between the coverage level and the expected loss rate. For example, suppose the published conventional planted rate was estimated as $30.20 \%$ (as a percentage of liability). If this were the rate charged on conventional production, then producers would pay a rate of $15.10 \%$ for insuring skip-row cropping. Yet, the expected loss cost for a skip-row producer in this case was only $6.40 \%$ (not $15.10 \%$ ), nearly $60 \%$ less than the expected loss cost of $15.10 \%$. Thus, not only was coverage availability severely restricted relative to conventional under the 2008 rules, but the rates were also severely inflated for any effective level of coverage for skip-row production.

In order to assess spatial patterns in the results, Figure 5 presents a map of the ratio of skip-row to conventionally-planted production expected loss costs for the $85 \%$ coverage level product when the conventionally-planted APH is employed for both. The simulated average unit-level loss costs are averaged together at the county level of aggregation for exposition. The figures suggest substantial heterogeneity in the relationship between skip-row and conventional insurance loss rates across the region. All of the counties have lower expected skip-row loss costs than their conventionally-planted counterparts when using the conventionally-planted APH (Figure 5). However, the expected difference is smaller in the high-yielding eastern region, as expected. This indicates that, (relative to a given level of bu./acre coverage) skip-row was always less risky by this metric. Some regions have expected positive rate differen- 


\section{Legend}

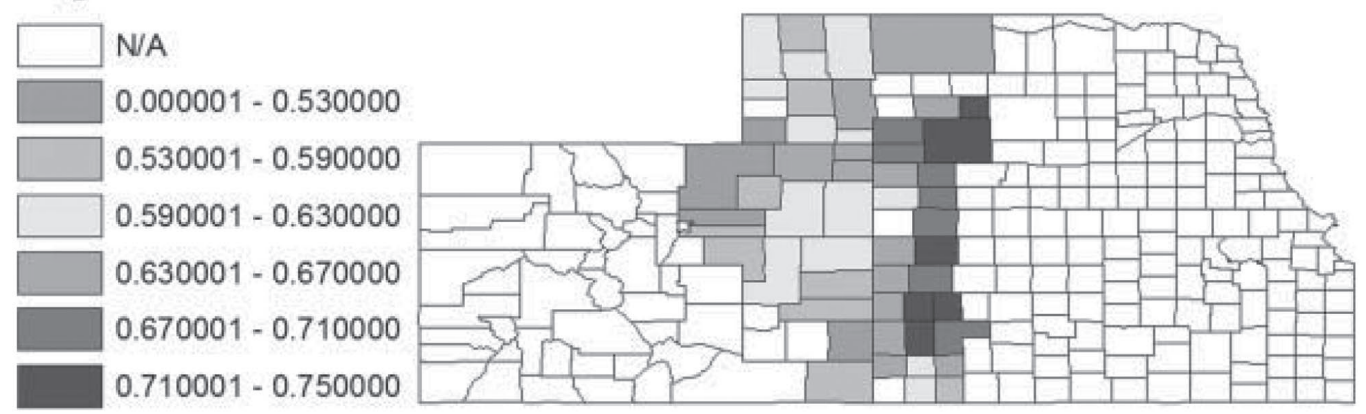

Figure 5. Ratio of Expected Loss Costs, Skip-Row to Conventional (Conventional APH, 85\% Coverage Level)

tials between skip-row and conventional expected loss costs, while others have negative differentials when using an adjusted skip-row APH for skip-row insurance (map not presented). This indicates that as APH's adjust to reflect skip-row average production capacity, the expected loss rates for skip-row and conventional production will not necessarily converge. The implication is that the 2009 rules will not result in equal expected loss rates for skip-row and conventionally-planted crops, even after APH's have adjusted. Thus, initially we would expect all regions to have significantly lower expected loss rates for skip-row cropping if the conventional APH is applied for both. Thus, combining both practices into the same insurance program as under the current rules is probably not warranted.

Overall, we find consistent evidence that adopting skip-row cropping will result in lower yield risk (relative to a given level of bu./acre coverage) and higher average yields. These results suggest that RMA should do one of two things, either (1) consider insuring skip-row and conventionally-planted crops separately, and maintain them as separate insurable practices (similar to how non-irrigated and irrigated practices are maintained separately), including keeping separate APH records, and maintaining separate rates and T-Yields, or (2) that RMA implement rate and APH corrections for the proportion of acreage in the insurable unit allocated to skip-row so that the effective coverage levels and rates charged reflect the actual production capabilities and risks of the practices appropriately. Failure to modify the current rules may incentivize producers to not adopt skip-row production in cases where they otherwise might if the provisions for insurance were fair across practices, or if no insurance existed. We investigate this further in the expected utility analysis in the next section.

\section{Results - Expected Utility Analysis and Technology Adoption}

Table 4 presents results for expected net returns, risk (LPM), and expected utility of returns for conventional and skip-row practices under various insurance regimes for a representative producer in Thomas, Kansas. Four scenarios are evaluated: no insurance, insurance under the 2008 rules, insurance under the current rules, and insurance under the proposed rules. Recall that net returns are calculated as the yield plus any insurance indemnities, minus insurance costs (net of premium subsidy), and results are presented for $85 \%$ coverage level insurance. Table 4 summarizes the expected utility maximizing technology choice (skip-row or conventional) in each case.

Under no insurance, skip-row has both a higher expected return and greater expected utility than conventional production, as well as lower risk. Under the 2008 rules, however, the insured skip-row net return had both a lower return and higher risk than an insured conventional crop. This reflects the fact that coverage was restricted for skip-row relative to conventional (and had an inflated premium) under those rules due to the use of FSA factoring, and also that the rules were inhibiting the adoption of skip-row production. Similar results are found under current insurance rules. While the insured risk for skip-row cropping is slightly lower than insured conventional, the expected return and expected utility are also lower, reflecting the fact that skip-row expected loss costs are lower than conventional; yet they receive the same premium rate as conventional cropping under current rules. This result was consistent for expected utility across all reasonable levels of risk aversion, and indicates that an expected utility maximizing producer would typically adopt conventional 
planting under current insurance rules. Last, under the proposed rules, insured skip-row planting has both higher expected utility and a higher expected return than conventional production, as well as lower risk. Thus, an expected utility maximizing producer would adopt skip-row under the proposed rules. We believe that the proposed rules would be the most actuarially appropriate, as the 2008 and current insurance rules were both shown to (1) incorrectly reflect skip-row production potential in APH's, and (2) overcharge for skip-row insurance relative to a given bu./acre level of coverage for conventional planting. The expected utility analysis also indicates that the new proposed rules would not disincentivize skiprow adoption, whereas the existing rules do.

\section{Conclusion}

This study investigates the performance of skiprow corn planting in the Central Great Plains by developing a multivariate nonparametric simulation technique that allows yield trial data to be efficiently combined with larger existing databases of yields in order to derive augmented skip-row yield distributions and insurance rates using the conditional distribution generated from the trial data. The results suggest that skip-row corn planting embodies both higher average productivity and lower risk in this region. The expected impacts of skip-row adoption on loss rates are quite large, with $100 \%$ differences between conventional and skip-row loss rates appearing to be typical. The implication is that skip-row corn in this region embodies an essentially different set of risks than conventional practices, suggesting that separate insurance programs for skip-row and conventional crops may be warranted. Overall, the results indicate that under the 2008 rules, and even under the current rules, skip-row adoption is likely to be crowded out due to the unfair provision of insurance across practices, whereas it otherwise would be optimal to adopt if no insurance were available, or if a coherent insurance program were in place.

We acknowledge that the implied insurance loss cost differences suggested by this study are large. While those presented are the best estimates of the actuarially fair rate differentials based on available data, and while it is conceivable that the actual rate adjustments may indeed be this large, we caution that they are based mainly on trial data-the 270 yield trial observations from Lyon et al. (2009)-and thus in practice may lack the proper credibility for a full and immediate rate adjustment. In practice, skip- 
row planting could initially employ the conventional planted insurance rate and $\mathrm{APH}$, along with modest rate and $\mathrm{APH}$ adjustments, which could be updated through time as more data become available. Nevertheless, the results of this analysis and other research appear to provide a sound basis for splitting skiprow and conventional planting practices into separate insurable practices. Thus, future research should focus on continued evaluation of skip-row performance in the Central Great Plains.

\section{References}

Atwood, J. S. Shaik, and M. Watts. 2003. Are Crop Yields Normally Distributed? A Reexamination. American Journal of Agricultural Economics 85(4):888-901.

Abunyewa, A. A. 2008. Efficient Utilization of Water and Nitrogen Resources for Grain Sorghum under Rainfed Conditions. PhD Dissertation, University of Nebraska.

Akerlof, G. A. 1970. The Market for "Lemons: Quality Uncertainty and the Market Mechanism." The Quarterly Journal of Economics 84(3): 488-500.

Arrow, K. J., "Uncertainty and the Welfare Economics of Medical Care," The American Economic Review, 53-5 (Dec., 1963): 941-973.

Bowman, A. W., and A. Azzalini, "Applied Smoothing Techniques for Data Analysis: The Kernel Approach with S-Plus Illustrations," Oxford University Press (1997).

Hölmstrom, B., "Moral Hazard and Observability," The Bell Journal of Economics, 10-1(Spring, 1979): 74-91.

Holt, C. A., and S. K. Laury. "Risk Aversion and Incentive Effects," The American Economic Review, 92-5(Dec. 2002):1644-55.

Jaffee, D., "Should the Government Provide Insurance for Catastrophes? Comments on a Paper by J. David Cummins," Federal Reserve Bank of St. Louis Review, 88-4 (July/ August 2006):337-379.
Lyon, D. J., A. D. Pavlista, G. W. Hergert, R. N. Klein," C. A. Shapiro, S. Knezevic, S. C. Mason, L. A. Nelson, D. D. Baltensperger, R. W. Elmore, M. F. Vigil, A. J. Schlegel, B. L. Olson, and R. M. Aiken, "Skiprow planting patterns stabilize corn grain yields in the Central Great Plains," Crop Management (2009).

Palic, D. B., P. Burgener, G. H. Dunn, M. S.Vigil, and A. D. Pavlista, "Analyzing Insurance Implications for Skip-Row Corn Using iFarm Economic Analysis Tool (iFEAT)," 20th Annual High Plains No-Till Conference (February 5-6, 2008).

Pavlista, A. D., D. J. Lyon, D. D. Baltensperger, and G. W. Hergert, "Yield Components as Affected by Planting Dryland maize in a Double-Skip Row Pattern," Journal of Crop Improvement, 24(2010):131-141.

Rothschild, M., and J. Stiglitz, "Equilibrium in Competitive Insurance Markets:An Essay on the Economics of Imperfect Information," The Quarterly Journal of Economics, 90-4(Nov., 1976): 629-649.

Simar, L. and P.W. Wilson, "Estimating and Bootstrapping Malmquist Indices," European Journal of Operational Research, 115(1999): 459-471.

Whish J., G. Butle, M. Castor, S. Cawthray, I. Broad, P. Carberry, G. Hammer, G. McLean, R. Routley, and S.Yeates,"Modeling the Effects of Row Configuration on Sorghum Yield in Northeastern Australia," Australian Journal of Agricultural Research 56(2005): 11-23.

Woodard, J. D., G. D. Schnitkey, and B. J. Sherrick, "Crop Insurance Ratemaking under Trending Liabilities," Journal of Agricultural and Resource Economics (2011).

Woodard, J. D., "Three Essays on Systemic Risk and Rating in Crop Insurance Markets," PhD Dissertation, University of Illinois at Urbana-Champaign (2008). Available online at: http://gradworks.umi. com $/ 33 / 37 / 3337964$.html

Woodard, J. D., G. D. Schnitkey, B. J Sherrick, N. Lozano-Gracia, and L. Anselin,"A Spatial Econometric Investigation of Loss Experience in the U.S. Crop Insurance Program," Journal of Risk and Insurance (2011). 